\title{
Establishment of human trophoblast stem cells from human induced pluripotent stem cell-derived cystic cells under micromesh culture
}

\author{
Zhuosi Li ${ }^{1 *}$ Osamu Kurosawa ${ }^{1}$ and Hiroo Iwata ${ }^{1,2}$
}

\begin{abstract}
Background: Trophoblasts as a specific cell lineage are crucial for the correct function of the placenta. Human trophoblast stem cells (hTSCs) are a proliferative population that can differentiate into syncytiotrophoblasts and extravillous cytotrophoblasts. Many studies have reported that chemical supplements induce the differentiation of trophoblasts from human induced pluripotent stem cells (hiPSCs). However, there have been no reports of the establishment of proliferative hTSCs from hiPSCs. Our previous report showed that culturing hiPSCs on micromesh as a bioscaffold induced cystic cells with trophoblast properties. Here, we aimed to establish hTSCs from hiPSCs.
\end{abstract}

Methods: We used the micromesh culture technique to induce hiPSC differentiation into trophoblast cysts. We then reseeded and purified cystic cells.

Results: The cells derived from the reseeded cysts were highly proliferative. Low expression levels of pluripotency genes and high expression levels of TSC-specific genes were detected in proliferative cells. The cells could be passaged, and further directional differentiation into syncytiotrophoblast- and extravillous cytotrophoblast-like cells was confirmed by marker expression and hormone secretion.

Conclusions: We established hiPSC-derived hTSCs, which may be applicable for studying the functions of trophoblasts and the placenta. Our experimental system may provide useful tools for understanding the pathogenesis of infertility owing to trophoblast defects in the future.

Keywords: Micromesh, Trophoblast, Human pluripotent stem cell, Syncytiotrophoblast, Extravillous trophoblast

\section{Background}

Trophoblasts play key roles in the placenta. Placentation starts with the formation of the trophectoderm in pre-implantation blastocysts. After implantation, trophectoderm cells differentiate into diverse trophoblast cell types. Mononuclear cytotrophoblasts (CTBs), derived from the trophectodermal layer, are considered to be trophoblast stem cells (TSCs) and differentiate into either syncytiotrophoblasts (STBs) or extravillous trophoblasts (EVTs) [1]. STBs comprise the cell layer that covers the outer surface

\footnotetext{
*Correspondence: zhuosi.li@riken.jp

${ }^{1}$ The "Compass to Healthy Life" Research Complex Program, RIKEN Institute, Kobe 650-0047, Japan

Full list of author information is available at the end of the article
}

of the villi and are responsible for nutrient and gas exchange in the human placenta [2]. Villous STBs are also the major site for the production of numerous hormones, such as human chorionic gonadotropin (hCG). For the maintenance of pregnancy and immunomodulation, hCG needs to be delivered to the maternal side and circulated in the body [3, 4]. TSCs differentiate into EVTs at villus/ uterus anchoring sites. EVTs begin to migrate into the decidual tissue or toward maternal blood vessels. This invasion causes maternal arteria to lose contractility and maintain sufficient maternal blood flow in intervillous spaces to support placental function [5]. Several reports have demonstrated that inadequate formation of the STB

(c) The Author(s). 2019 Open Access This article is distributed under the terms of the Creative Commons Attribution 4.0 International License (http://creativecommons.org/licenses/by/4.0/), which permits unrestricted use, distribution, and 
structure or failure of EVT invasion leads to severe pregnancy disorders, such as preeclampsia [6].

Various cell types have been used in placental research. Human choriocarcinoma cell lines, such as BeWo and JEG-3, have been widely used as models for mimicking in vitro trophoblast cell fusion in the presence of cyclic adenosine monophosphate (cAMP)-elevating agents, such as forskolin or 8-bromo-cAMP [7, 8]. Primary cells isolated and purified from the human term placenta have been used to obtain functional STBs [9]. Okae et al. [10] have successfully isolated and maintained human TSC (hTSC) lines from first-trimester placentas and have shown that these hTSCs have the capacity to differentiate into STBs and EVTs. However, because of ethical problems, it is difficult to acquire human placental tissues and blastocysts in order to isolate cells.

According to recent reports, human induced pluripotent stem cells (hiPSCs) and human embryonic stem cells (hESCs) can be induced into trophoblast lines using culture medium with added bone morphogenetic protein 4 (BMP4) and without fibroblast growth factor-2 (FGF2) [11-14]. BMP4 has been reported to induce CTBs that cannot proliferate but can nondirectionally differentiate into STBs and EVTs in the same culture system [15].

Bioscaffolds, such as collagen, silk, and nanofibers, are increasingly used to promote the differentiation of hiPSCs and the maintenance of cellular physiological functions $[16,17]$. We originally developed a cell culture technique with a microstructured mesh sheet as a bioscaffold. The apertures of micromeshes commonly used for culture are much larger in size than single cells, allowing cells present at the openings to become to be bound only by cell-cell adhesion [18]. Minimization of cell-basal substrate adhesion can promote cellular functions and is beneficial for cells to obtain oxygen and nutrients. Notably, micromesh-cultured hepatic cells have been shown to exhibit increased expression of hepatic marker genes and enhanced cell maturation [18]. Furthermore, Okeyo et al. [19] reported that micromesh culture induces spontaneous differentiation of hiPSCs into trophoblast cyst structures without BMP4 treatment.

In the current study, we aimed to establish hiPSC-derived TSCs using the micromesh culture method without BMP4 treatment. We confirmed the differentiation of hiPSC-derived trophoblast cells under micromesh culture conditions and examined whether the cells could differentiate into STBs and EVTs. We hypothesized that micromesh culture techniques may have great potential to yield TSCs from the hiPSCs of patients with infertility or genetic diseases and could facilitate the analysis of trophoblast functions in both normal and diseased pregnancies in vitro. Our findings may establish a potential model for understanding the differentiation and function of trophoblasts and may yield further information regarding the pathogenesis of infertility associated with trophoblast defects for clinical application.

\section{Methods \\ hiPSC culture and differentiation}

The ChiPSC22 hiPSC line (Takara Bio, Kusatsu, Japan) was cultured in Cellartis DEF-CS 500 culture medium (Takara Bio) according to the manufacturer's protocols. Briefly, cells were passaged with TrypLE reagent (Gibco/ Life Technologies, Carlsbad, CA, USA) every 3-4 days, and the culture medium was replaced daily. Only passages $10-20$ were used for all experiments described here.

Differentiation of trophoblasts was performed using the micromesh culture method. Briefly, a nickel micromesh with triangular shapes (Optnics Precision Co., Ltd., Ashikaga, Japan) was obtained. The side length of the triangle was $50 \mu \mathrm{m}$, the thickness of the sheet was $5 \mu \mathrm{m}$, and the width of the mesh strand was $5 \mu \mathrm{m}$ (Fig. $1 \mathrm{~A}-\mathrm{a}, \mathrm{b}$ ). The micromesh was cut into $0.6 \times 0.6 \mathrm{~cm}$ sections and fixed on a silicon sheet $(1 \times 0.5 \times 1 \mathrm{~cm}$, length $\times$ height $\times$ width $)$ using a Kapton tape (Fig. 1A-a). The silicon sheet and Kapton tape had a 4-mm hole in the center to act as a frame reinforcing the ultrathin mesh sheets (Fig. 1A-a). The mesh was coated with a thin layer of parylene in a PDS2010 system (Specialty Coating Systems) and was then air plasma treated using a plasma cleaner (PDC-001HP; Harrick Plasma) at a high radio frequency for $3 \mathrm{~min}$. The reinforced mesh sheets were sterilized using ultraviolet irradiation for $90 \mathrm{~min}$. Following sterilization, $50 \mu \mathrm{L}$ of $3.2 \mu \mathrm{g} / \mathrm{mL}$ iMatrix-511 (Takara Bio) was applied to the hole of the mesh sheet and allowed to coat the hole at $37^{\circ} \mathrm{C}$ for at least $1 \mathrm{~h}$. Next, $50 \mu \mathrm{L}$ of an hiPSC suspension $\left(2 \times 10^{6}\right.$ cells $\left./ \mathrm{mL}\right)$ was gently pipetted into the hole of the mesh sheet (Fig. $1 \mathrm{~A}-\mathrm{C}$ ). After incubation at $37^{\circ} \mathrm{C}$ for $4 \mathrm{~h}$, the mesh sheets were suspended in culture medium using a $0.5-\mathrm{cm}$-thick silicon spacer such that the cells seeded on the mesh sheets were completely out of contact with the dish bottom (Fig. 1A-d, e). hiPSCs were cultured on a mesh sheet at $37^{\circ} \mathrm{C}$ in an atmosphere of $5 \% \mathrm{CO}_{2}$ in DEFCS medium for approximately 30-50 days, with the culture medium replaced every 3 days.

\section{TSC culture}

After 30-50 days, trophoblast cysts were observed in the mesh culture. The cysts were separated from the mesh using microscissors or a microscalpel (Additional file 3: Movie S1) and transferred to iMatrix511-coated 24-well plates. Each cyst was seeded in a single well. For proliferation of cystic trophoblasts on the bottom of the plate, the basic culture medium for hTSC reported by Okae et al. [10] was used. Briefly, the cysts were cultured in hTSC medium (Dulbecco's 


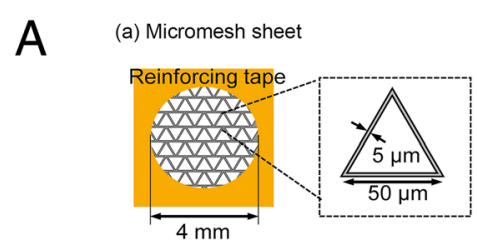

(c) Bird's eye view

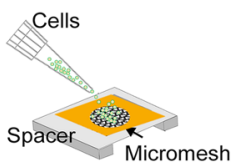

(b)

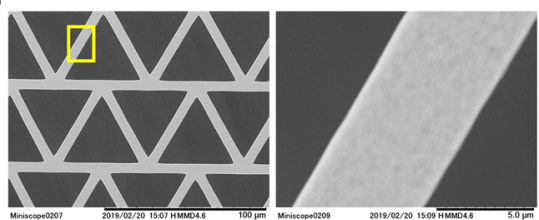

(d) Side view

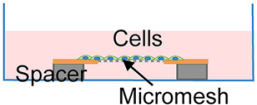

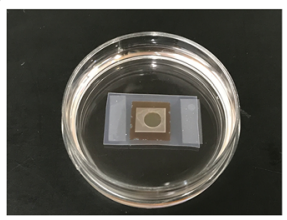

B

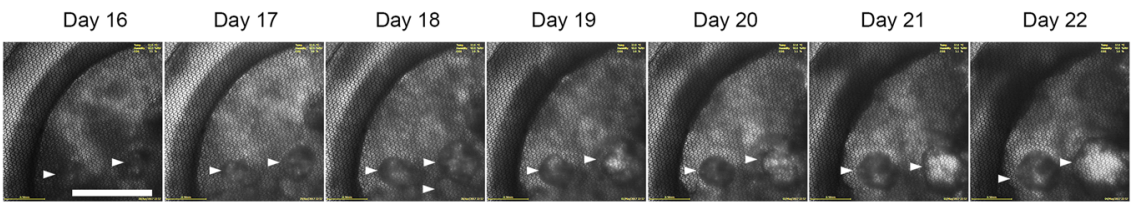

C

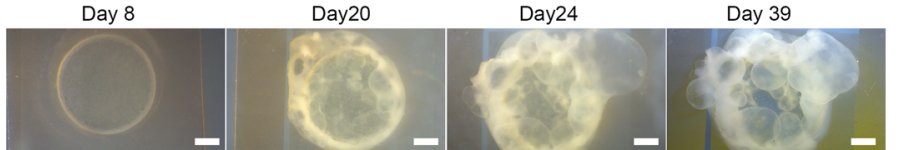

D

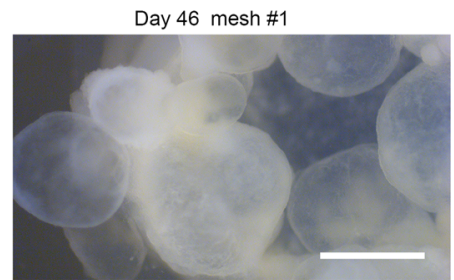

F

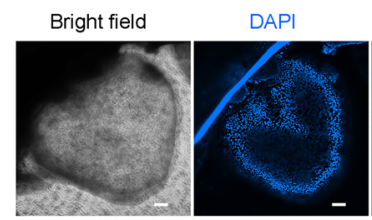

$\mathrm{hCG}$

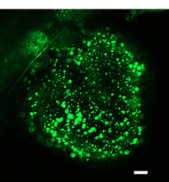

E

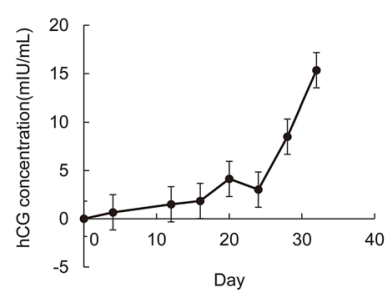

DAPI hCG

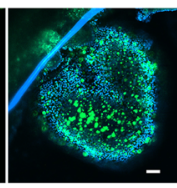

Merge

3D
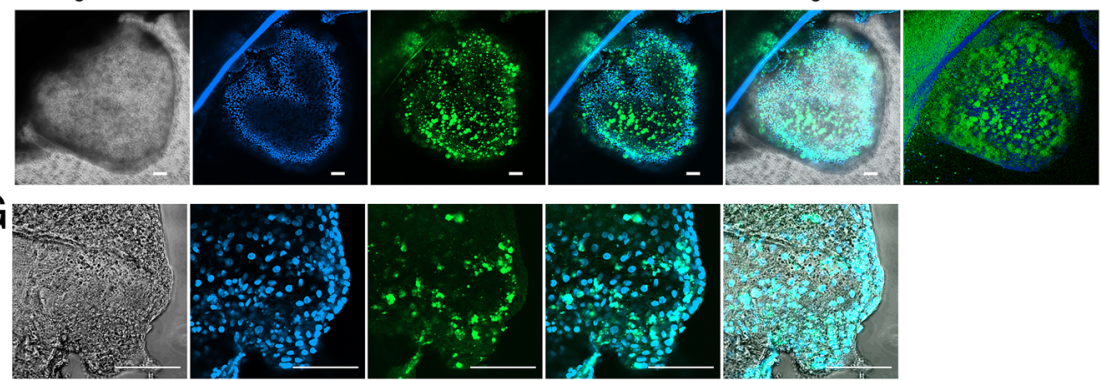

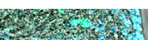
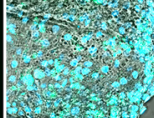

in $15,2,3\}$

Fig. 1 Induction of a trophoblastic cyst structure in hiPSCs in a micromesh culture. A Schematic diagram showing the procedures for cell seeding and culture in a limited area on a micromesh. (a) Mesh shapes and dimensions. (b) Scanning electron microscope image of a fabricated triangular mesh. (c, d) Schematic showing how cell seeding and culture were performed on mesh sheets suspended in a culture medium. (e) Real product photograph. A micromesh sheet seeded with hiPSCs was placed in a 3.5-cm dish and cultured for 30-50 days. B Time-lapse monitoring of hiPSCs on a 4-mm suspended mesh sheet. Phase-contrast images of hiPSCs on days 16-22 of micromesh culture. Scale bar = 1 mm. $\mathbf{C}$ Stereomicroscopic images of hiPSCs in micromesh culture. Scale bar $=1 \mathrm{~mm}$. D Enlarged view of stereomicroscope images, showing numerous cysts in the micromesh culture on day 46. E Quantitative analysis of hCG secretion by ELISA. Culture supernatants were collected from micromesh culture samples. Data are presented as means \pm SEMs $(n=4)$. F Immunofluorescence analysis of cysts. Bright-field and immunofluorescence images of representative cysts stained for hCG. Nuclei were stained with DAPI (blue). Scale bar = $100 \mu$ m. G High-magnification images of immunofluorescence for hCG. To scan high-magnification images using a confocal microscope, individual cysts were resected and placed on the cover glass for observation. Scale bar $=100 \mu \mathrm{m}$ 
modified Eagle's medium [DMEM]/F12 medium supplemented with $0.1 \mathrm{mM}$ 2-mercaptoethanol, $0.2 \%$ fetal bovine serum, $1 \%$ penicillin/streptomycin, $0.3 \%$ bovine serum albumin [BSA], 1\% ITS-X supplement, $1.5 \mathrm{mg} / \mathrm{mL}$-ascorbic acid, $50 \mathrm{ng} / \mathrm{mL}$ epidermal growth factor [EGF], $2 \mathrm{mM}$ CHIR99021, $0.5 \mathrm{mM}$ A83-01, $1 \mathrm{mM}$ SB431542, $0.8 \mathrm{mM}$ valproic acid, and $5 \mathrm{mM}$ Y27632). The culture medium was replaced every 2 days.

Ten days after cyst seeding, cells proliferating from cysts were collected and passaged. The cells were characterized as TSCs. For passaging, these TSCs were seeded at a density of $2.5 \times 10^{5}$ cells/well in 6-well plates, which was precoated with $1.5 \mathrm{~mL}$ of $3.2 \mu \mathrm{g} / \mathrm{mL}$ iMatrix511, and cultured in 2-mL hTSC medium for 3-4 days, with culture medium replaced every 2 days. At $70-80 \%$ confluence, the cells were dissociated with TrypLE and Accumax (1:1) for $6 \mathrm{~min}$ at $37^{\circ} \mathrm{C}$ and then reseeded and cultured in an hTSC medium. The cells initially grew slowly but then yielded highly proliferative cells within several passages. Thereafter, trophoblast cells were routinely passaged every 3 days at a 1:4 split ratio. For cryopreservation, trophoblast cells were suspended in Cell Banker 1 and stored in a deep freezer at $-80^{\circ} \mathrm{C}$ or in liquid nitrogen.

\section{TSC differentiation}

For differentiation of TSCs into EVTs and STBs, a procedure described by Okae et al. [10] was used. Briefly, TSCs were grown to $80 \%$ confluence in hTSC medium and dissociated with TrypLE and Accumax (1:1) for $6 \mathrm{~min}$ at $37^{\circ} \mathrm{C}$. For induction of three-dimensional (3D) STB cells, TSCs were seeded in low-adhesion Petri dishes at a density of $2.5 \times 10^{5}$ cells/well and cultured in 3-mL STB-(3D) medium (DMEM/F12 supplemented with $0.1 \mathrm{mM}$ 2-mercaptoethanol, $0.5 \%$ penicillin/streptomycin, $0.3 \%$ BSA, $1 \%$ ITS-X supplement, $2.5 \mathrm{mM}$ Y27632, $50 \mathrm{ng} / \mathrm{mL}$ EGF, $2 \mathrm{mM}$ forskolin, and $4 \%$ knockout serum replacement [KSR]). An equal volume of fresh STB-(3D) medium was added on day 3, and the cells were analyzed on day 6 .

For induction of EVTs, TSCs were seeded at a density of $0.75 \times 10^{5}$ cells/well in 6-well plates precoated with $1 \mathrm{mg} / \mathrm{mL}$ collagen IV and then cultured in 2-mL EVT medium (DMEM/F12 supplemented with $0.1 \mathrm{mM}$ 2-mercaptoethanol, $0.5 \%$ penicillin/streptomycin, $0.3 \%$ BSA, $1 \%$ ITS-X supplement, $100 \mathrm{ng} / \mathrm{mL}$ human neuregulin-1 [NRG1], 7.5 mM A83-01, $2.5 \mathrm{mM}$ Y27632, and 4\% KSR). Matrigel was added to a final concentration of $2 \%$ shortly after suspending the cells in the medium. On day 3, the medium was replaced with EVT medium without NRG1, and Matrigel was added to a final concentration of $0.5 \%$ [10].

\section{Immunostaining}

Cells were fixed with $4 \%$ paraformaldehyde (PFA) for 15 min, permeabilized with $0.1 \%$ Triton X-100 for 10 min, and blocked with Blocking One buffer (Nacalai Tesque, Kyoto, Japan) for $1 \mathrm{~h}$ at room temperature. The cells were then incubated with primary antibodies overnight at $4{ }^{\circ} \mathrm{C}$. The following primary antibodies were used: anti-hCG (cat. no. ab9582; 1:100; Abcam, Cambridge, UK), anti-E-cadherin (cat. no. 3195; 1:100; Cell Signaling Technology, Danvers, MA, USA), anti-tumor protein p63 (TP63; cat. no. 13109; 1:100; Cell Signaling Technology), anti-SRY (sex determining region Y)-box 2 (SOX2; cat. no. 3579S; 1:400; Cell Signaling Technology), antiNanog homeobox (NANOG; cat. no. 4903S; 1:200; Cell Signaling Technology), anti-keratin 7 (KRT7; cat. no. 15539-1-AP; 1:200; Proteintech, USA), and anti-major histocompatibility complex, class I, G (HLA-G; cat. no. 4H84; 1:100; Abcam), anti-POU class 5 homeobox 1 (POU5F1; cat. no. 75463; 1:200; Cell Signaling Technology), and anti-GATA-binding protein 3 (GATA3; cat. no. 96098; 1:100; Cell Signaling Technology). Finally, cells were incubated with Alexa Fluor 488- or Alexa Fluor 594-conjugated secondary antibodies (1:500; Invitrogen, Carlsbad, CA, USA). All antibodies were diluted in phosphate-buffered saline (PBS) containing 1\% BSA. For hiPSCs, the undifferentiation staining lectin marker rBC2LCN-488 (100-fold diluted; Wako Pure Chemical Industries, Ltd., Osaka, Japan) was mixed with medium, and hiPSCs were incubated in this medium for $2 \mathrm{~h}$ at $37^{\circ} \mathrm{C}$ and $5 \% \mathrm{CO}_{2}$ for labeling. Nuclei were stained with 4',6-diamidino-2-phenylindole (DAPI) or Hoechst 33342, and images were acquired with a laser scanning confocal microscope (LSM 800; Carl Zeiss, Oberkochen, Germany).

\section{Analysis of hCG}

Aliquots of medium were collected for enzyme-linked immunosorbent assay (ELISA) analysis from cultures with hiPSC-derived cysts and stored at $-20^{\circ} \mathrm{C}$. Secreted hCG was assayed using an hCG ELISA kit (Immunospec, Canoga Park, CA, USA) according to the manufacturer's instructions.

\section{Real-time quantitative polymerase chain reaction (qRT- PCR) analysis}

Cells plated in 24-well plates (5,000 cells/well) or hiPSCderived cysts were collected, and qRT-PCR was performed using a Power SYBR Green Cells-to-CT kit, as described by the manufacturer (Invitrogen). The expression level of each gene was normalized to that of glyceraldehyde 3phosphate dehydrogenase (GAPDH) used as an internal control. Representative data from three independent experiments are shown. The primers used for qRT-PCR are shown in Additional file 1: Table S1. 


\section{Flow cytometry}

Cells were dissociated with TrypLE and Accumax (1:1) for $6 \mathrm{~min}$ at $37^{\circ} \mathrm{C}$ and resuspended in PBS. For flow cytometric analysis of KRT7, cells were fixed with 4\% PFA for $15 \mathrm{~min}$ at room temperature and permeabilized with $0.1 \%$ Triton X-100 for $10 \mathrm{~min}$. The cells were then incubated with anti-KRT7 antibodies (1:100) overnight at $4{ }^{\circ} \mathrm{C}$ and stained with Alexa Fluor 488-conjugated antirabbit IgG for $1 \mathrm{~h}$ at $25^{\circ} \mathrm{C}$. Normal rabbit IgG was used as an isotype control. Flow cytometry was carried out using an SH800 cell sorter (Sony, Tokyo, Japan), and the data were analyzed using SH800 software.

\section{mRNA expression array analysis}

For mRNA microarray analysis, 3D-Gene Human Oligo Chip $25 \mathrm{~K}$ (Toray Industries, Inc., Tokyo, Japan) was used. Total RNA was amplified using an amino allyl aRNA kit (Ambion, Inc., Foster City, CA, USA). The total RNA was labeled with cyanine 5 (Cy5) using Amersham Cy5 mono-reactive dye (GE Healthcare, Little Chalfont, Buckinghamshire, UK). Cy5-labeled aRNAs were individually hybridized at $37^{\circ} \mathrm{C}$ for $16 \mathrm{~h}$. The chips were washed and scanned using a 3D-Gene scanner 3000 (Toray Industries, Inc.) and analyzed using 3DGene extraction software (Toray Industries, Inc.). The signals detected for each gene were subjected to global normalization (the median of the detected signal intensity was adjusted to 25$)$.

\section{Statistical analysis}

Data are presented as means \pm standard errors of the means (SEMs). Statistical analyses were conducted with SPSS Statistics version 22 (IBM, Armonk, NY, USA). Student's $t$ tests were used to determine the significance of differences, which was defined as $P<0.05$. For changes in hCG secretion by TS ${ }^{\text {hiPSC }}$ cells, one-way analysis of variance with repeated measures was conducted.

\section{Results}

Identification of hCG-positive trophectoderm-like cysts derived from hiPSCs in micromesh culture

Micromesh scaffolds have been used for hiPSC differentiation into trophectoderm-like cysts $[19,20]$. Consistent with previously reported data, our data showed that cystic structures emerged on day 16 when using a $4-\mathrm{mm}$ nickel micromesh (Fig. 1B). With continuous culture, the cysts grew to a diameter of $\sim 2 \mathrm{~mm}$ (Fig. 1B-D).

We then assayed the secretion of hCG, a key pregnancy hormone secreted by placental trophoblasts [21], using ELISAs. The levels of hCG markedly increased in the cyst-containing medium after day 15 (Fig. 1E) and continued to increase up to day 35. The localization of hCG in the cysts was examined by immunostaining, and the results revealed the presence of hCG in the cytoplasm of cyst-forming cells, providing evidence for hiPSC differentiation into cystic trophoblast cells in the micromesh culture (Fig. 1F, G).

\section{Generation of trophoblast stem-like cells from hiPSC- derived cysts}

We then analyzed the expression of key pluripotency genes and trophoblast lineage-specific genes in trophectoderm-like cysts using qRT-PCR analysis. The expression levels of POU5F1 and SOX2 significantly decreased in cysts compared with that in hiPSCs (Fig. 2a). Moreover, NANOG was weakly expressed in the collected cysts, potentially because of the presence of undifferentiated cells.

Expression levels of STB-specific markers, including chorionic gonadotropin subunit beta 3 (CGB3) and Kruppel-like factor 4 (KLF4), significantly increased in cysts compared with those in hiPSCs (Fig. 2a). Expression levels of hTSC markers, including TP63, KRT7, and E74-like ETS transcription factor 5 (ELF5), significantly increased in cysts compared with those in hiPSCs. ATP-binding cassette subfamily $B$ member 1 (ABCB1) is a widely studied drug efflux transporter in the placental barrier, and its mRNA expression was markedly increased in the cysts compared with those in hiPSCs, indicating the presence of STBs. Caudal type homeobox $2(C D X 2)$ is functional in trophectoderm cells, and its mRNA expression increased 8 -fold in cysts compared with that in hiPSCs $(P=0.7)$. Gene expression of MYC proto-oncogene (MYC) increased 1.4-fold in cysts compared with that in hiPSCs $(P=0.1$; Fig. 2a). TEA domain transcription factor 4 , frizzled class receptor 5 , integrin subunit alpha 6 , and lowdensity lipoprotein receptor-related protein 5 play important roles in the formation and maintenance of hTSCs, and their gene expression levels did not increase in the cysts compared with those in hiPSCs. These results demonstrated that hiPSC-derived trophoblast cysts may contain different types of cells, including STBs, TSCs, and undifferentiated hiPSCs.

Next, the localization of E-cadherin was analyzed in hiPSC-derived cysts by immunostaining. We found that E-cadherin was clearly expressed at the interfaces between mononuclear cells (Fig. 2b). Nuclear staining of cysts with DAPI showed that several cells were undergoing cell division (Fig. 2c). Based on these results, we attempted to obtain proliferating and mononuclear cells from hiPSC-derived cysts.

Cysts were separated and reseeded into cell culture dishes, and cells differentiating from cysts showed growth capacity (Fig. 2d). Because cysts contained several types of cells, we removed the cyst portion of the aggregates from the culture, and cells proliferating from the cysts (red-dotted box, Fig. 2d) were collected on day 3 for reseeding. The collected cells 
A
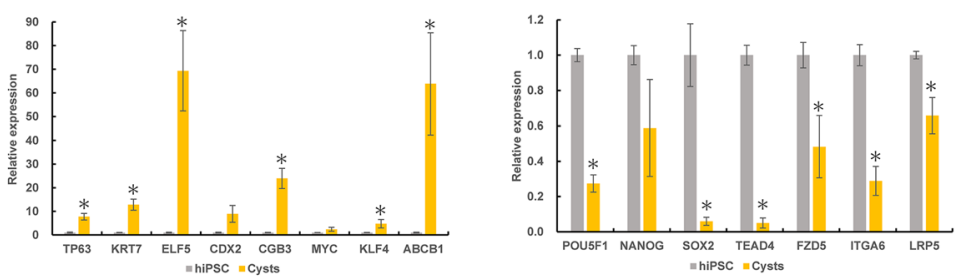

B

E-cadherin DAPI

\section{C}
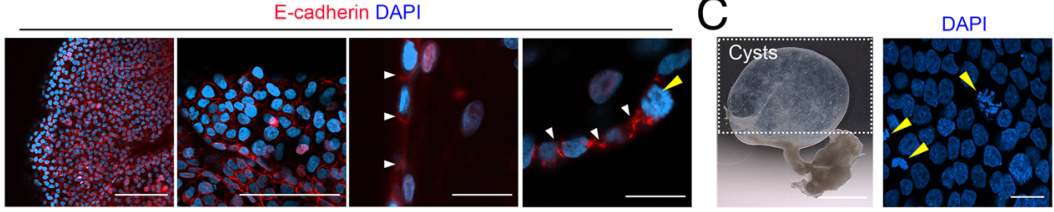

D

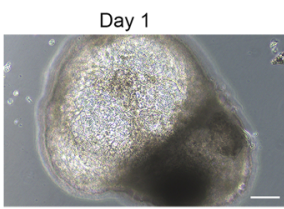

Day 2

Day 3

Day 5
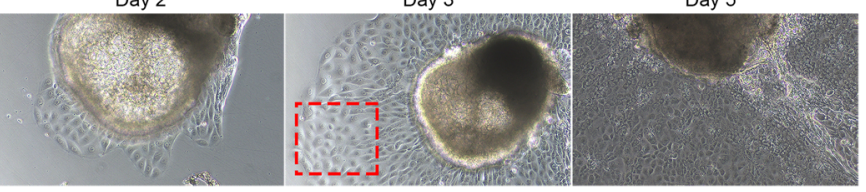

$E$

Day 0 (P14)

Day 2 (P14)

Day 4 (P14)

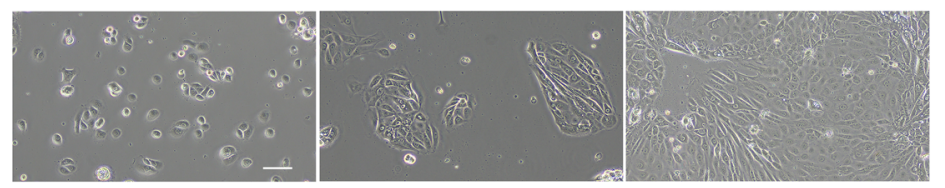

$\mathrm{F}$
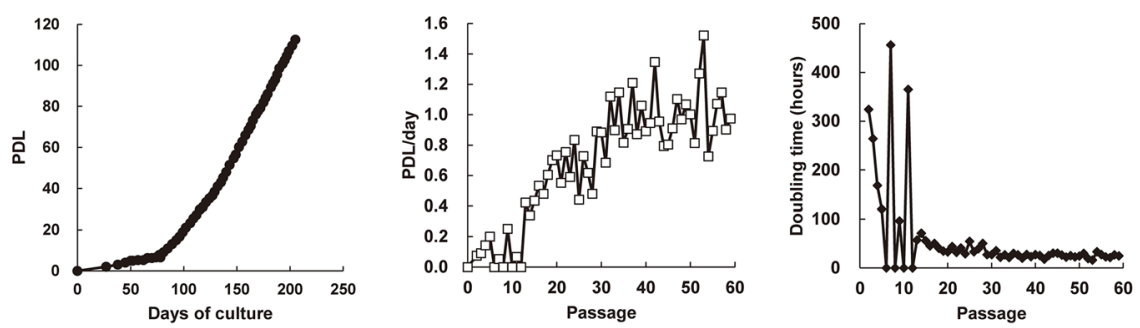

Fig. 2 Establishment of proliferative trophoblast cells from hiPSC-induced cysts. a Analysis of pluripotency and trophoblast gene expression by qRT-PCR in hiPSCs and cysts. Cysts were collected on day 46, and hiPSCs were grown on laminin-coated dishes for 3 days. Expression levels were calculated relative to those of GAPDH and normalized to those of control hiPSCs. Values are the means \pm SEMs $(n=4)$. Significance of differences was determined by Student's $t$ tests. ${ }^{*} P<0.05$ versus the hiPSC group. $\mathbf{b}$ Immunofluorescence images of a representative cyst stained for $E-$ cadherin. Scale bar $=100 \mu \mathrm{m}$ (two left images) and $20 \mu \mathrm{m}$ (two right enlarged images). Similar results were obtained for three independent samples from separate dishes. c Stereomicroscope images of separated cysts from 4-mm micromesh culture on day 46. Left: scale bar $=1 \mathrm{~mm}$. Right: nuclei were stained with DAPI (blue). Scale bar $=20 \mu \mathrm{m}$. d Phase-contrast images of time-lapse monitoring of a single cyst on days 1-5. The cyst was isolated from the micromesh culture and transferred to 24-well plates on day 0 . Cells derived from cysts in the area within the reddotted box were recovered for reseeding of trophoblast cells. Scale bar $=100 \mu \mathrm{m}$. e Phase-contrast images showing the growth of trophoblast cells derived from cysts after 14 passages. Scale bar $=100 \mu \mathrm{m}$. f Proliferation rate of trophoblast cells derived from cysts. Left, population doubling levels (PDLs) versus days of culture; middle, PDL/day versus passage number; and right, doubling time $(\mathrm{h})$ versus passage number

continued to proliferate in the hTSC medium and could be propagated for at least 59 passages, with 113 population doublings over 205 days (Fig. 2e, f). During the first 10 passages, the cells initially grew slowly; however, after several passages, the cells gave rise to highly proliferative cells, with a doubling time of 20-35 h (Fig. 2f). These proliferative trophoblasts were designated hiPSC-derived TSCs $\left(\mathrm{TS}^{\text {hiPSC}}\right)$ because they had the ability to differentiate into EVTand STB-like cells, as detailed below.

\section{Establishment of TSCs from hiPSC-induced cysts (TS ${ }^{\text {hiPSC }}$ )}

We next characterized $\mathrm{TS}^{\text {hiPSC }}$ cells as undifferentiated CTBs. TS ${ }^{\text {hiPSC }}$ cells showed a different cell morphology from hiPSCs (Fig. 3a). Compared with undifferentiated hiPSCs, TS ${ }^{\text {hiPSC }}$ cells showed loss of the pluripotency 

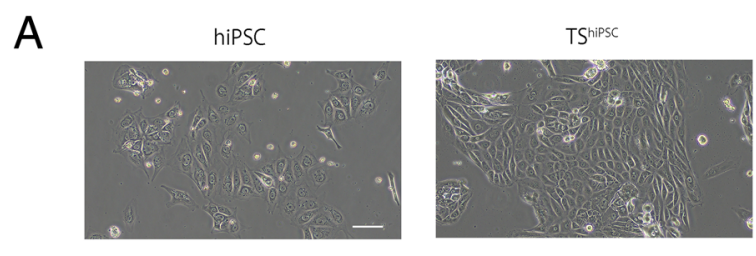

B
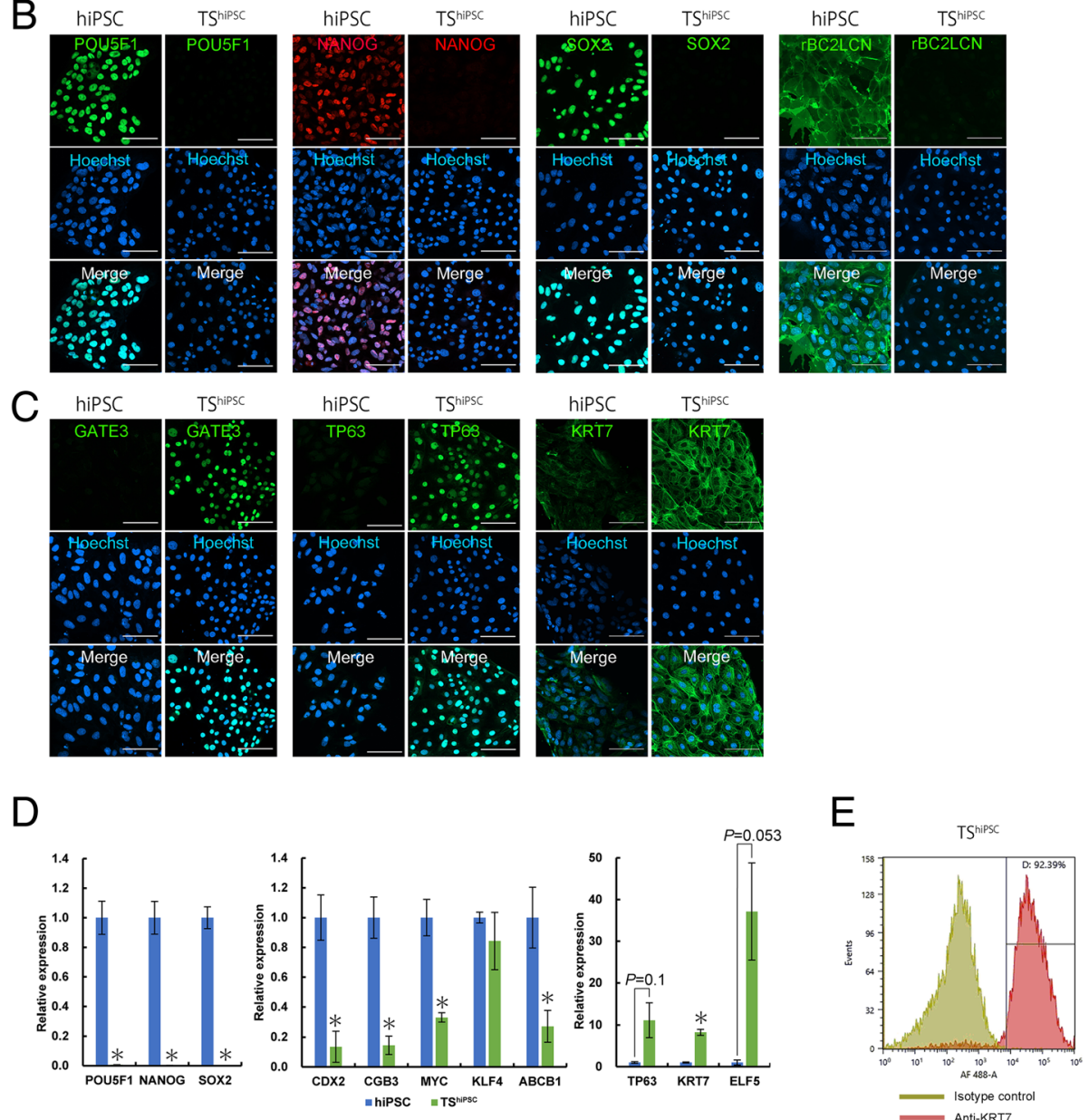

Fig. 3 Morphology of and marker expression in hiPSCs and trophoblast cells derived from cysts (TS ${ }^{\text {hiPSC}}$ ). a Phase-contrast images of hiPSCs and TS $^{\text {hiPSC }}$ cells. Scale bar $=100 \mu \mathrm{m}$. b, c Bright-field and immunofluorescence images of hiPSCs and TS ${ }^{\text {hiPSC }}$ cells stained for POU5F1, NANOG, SOX2, and rBC2LCN (b) and GATA3, TP63, and KRT7 (c). Nuclei were stained with Hoechst 33342. Scale bar $=100 \mu \mathrm{m}$. d Analysis of pluripotency and trophoblast gene expression by qRT-PCR in hiPSCs and TShiPSC cells grown on laminin-coated dishes for 3 days. Expression levels were calculated relative to those of GAPDH and normalized to those of control hiPSCs. Values are the means \pm SEMs $(n=4)$. Significance of differences was determined by Student's $t$ tests. ${ }^{*} P<0.05$ versus the hiPSC group. e Flow cytometry histograms of KRT7 expression in TS hiPSC cells. Similar results were obtained for three independent cell samples

markers NANOG, SOX2, and rBC2LCN (Fig. 3b); in contrast, the hTSC-associated proteins GATA3, TP63, and KRT7 were all highly expressed in $\mathrm{TS}^{\text {hiPSC }}$ cells (Fig. 3c). The results of qRT-PCR analysis showed that there was almost no expression of POU5F1, NANOG, and SOX2 in TS ${ }^{\text {hiPSC }}$ cells, whereas TP63 and KRT7 expression levels were significantly increased in TS ${ }^{\text {hiPSC }}$ cells compared with those in hiPSCs (Fig. 3d). In particular, the TSC-associated marker ELF5 was induced by 36-fold in TS ${ }^{\text {hiPSC }}$ cells (Fig. 3d). The STB markers
CGB3, MYC, KLF4, and $A B C B 1$ decreased in $\mathrm{TS}^{\mathrm{hiPSC}}$ cells (Fig. 3d). The level of cell purity was assessed by measuring the intracellular expression of the pantrophoblast marker KRT7 (Fig. 3e). The purity of KRT7positive cells was greater than $90 \%$ (Fig. 3e). These data indicated that cells derived from hiPSC-induced cysts assumed an hTSC phenotype. Furthermore, hTSC-associated marker expression in TS ${ }^{\text {hiPSC }}$ cells after several passages was assessed by immunostaining and qRT-PCR analysis. The hTSC-associated proteins KRT7, TP63, and 
GATA3 were all highly expressed in TS ${ }^{\text {hiPSC }}$ cells after $15,25,35$, and 55 passages (Additional file 4: Figure S1A). The results of qRT-PCR analysis showed that the genes TP63 GATE3, KRT7, and ELF5 were also all highly expressed in $\mathrm{TS}^{\text {hiPSC }}$ cells after 15, 25, 35, 45, and 55 passages compared with those in hiPSCs (Additional file 4: Figure S1B). The expression levels of these hTSC-associated markers were sustained after several passages.

\section{Global gene expression profiling of hiPSCs and TS ${ }^{\text {hiPSC }}$ cells}

To improve our understanding of the molecular characteristics of TS ${ }^{\text {hiPSC }}$ cells, we used a microarray approach, with hiPSCs as a control group. Trophoblast- and hiPSCrelated genes are shown in Fig. 4, and the entire data set and full gene names are included in Additional file 2: Table S2. Compared with their expression in hiPSCs, pluripotency factors, including $A F P, D N M T 3 B, D P P A 2$, DPPA4, FGF2, FOXA2, GDF3, GRB7, LEFTY2, LIN28B, NANOG, POU5F1, SOX2, ZFP42, and ZIC2, were downregulated in $\mathrm{TS}^{\text {hiPSC }}$ cells.

Genes responsible for trophoblast differentiation and function were expected to be upregulated in $\mathrm{TS}^{\text {hiPSC }}$ cells. Several genes upregulated in Fig. 4 have previously been shown to be involved in trophoblast development, such as genes encoding collagens (COL4A1 and COL4A2), keratins (KRT7 and KRT18), calcium-binding proteins (S100A11 and S100P), a transporter (SLC40A1), hormones (CGA and $C G B)$, and components of the extracellular matrix (COL3A1 and NID2). Others included the GATA3 gene, which is an important in vivo regulator of trophoblast-specific gene expression and placental function [22]; the $H S D 3 B 1$ gene, which is necessary for the biosynthesis of placental progesterone and is thus essential for pregnancy maintenance [23]; the placenta-specific IGF2 gene, which is a major modulator of placental and fetal growth [24]; and the EPAS1 gene, which is a hypoxia-responsive transcription factor involved in the regulation of endothelial cell gene expression [25]. KRT7, GATA3, and TFAP2C, which are good markers for first-trimester mononuclear trophoblast cells [26], were upregulated in $\mathrm{TS}^{\text {hiPSC }}$ cells. CDKN1C, CSF2RA, CYP11A1, EFHD1, FOLR1, HTRA4, ISM2, LVRN, PAGE4, and TFAP2C genes, which are expressed in primary CTBs from second-trimester placentas [27], were also upregulated in TS ${ }^{\text {hiPSC }}$ cells compared with those in hiPSCs. Moreover, microarray analysis confirmed the changes in gene expression profiles, as revealed by qRT-PCR analysis (Fig. 3d).

In this study, TS ${ }^{\text {hiPSC }}$ cells were induced from hiPSCs in a micromesh culture without BMP treatment. However, several genes associated with the emergence of trophoblast cells from BMP-treated hESCs, such as HAND1,
DLX3, CGA, CGB, IGF2, CYP11A1, HSD17B1, COL4A1, COL4A2, KRT7, KRT18, MMP2, TIMP3, S100A11, S100P, SLC4OA1, PPARG, and HOPX [28-30], were upregulated in $\mathrm{TS}^{\text {hiPSC }}$ cells. Therefore, we also evaluated the transforming growth factor- $\beta$ superfamily signaling network, including the BMP/GDF and ACTIVIN/NODAL branches. Inhibition of ACTIVIN/NODAL signaling and activation of BMP signaling are required for trophoblast differentiation from hESCs [31]. The microarray analysis showed systematic activation of directly inducible BMP target genes (DLX3, GATA3, HEY1, and MSX1). Genes encoding ligands and receptors supporting BMP signaling (BMP4, BMP5, and BMP7) were also induced. In contrast, genes encoding ACTIVIN/NODAL ligands (FGF2, FGF4, and $N O D A L$ ) [31] were downregulated. Similarly, the GDF3 gene, a known pluripotency-associated marker, acting via the initial signaling proteins SMAD2/3 of ACTIVIN/NODAL branches, was downregulated. Taken together, these gene expression pattern results provided reliable evidence for the characterization of TS ${ }^{\text {hiPSC }}$ cells as assumptive hTSCs.

\section{Differentiation capacity of TS ${ }^{\text {hiPSC }}$ cells}

To determine whether $\mathrm{TS}^{\text {hiPSC }}$ cells were multipotent, we investigated their ability to terminally differentiate into both STBs and EVTs. Some TS ${ }^{\text {hiPSC }}$ cells cultured in the hTSC medium spontaneously fused to form syncytia (Fig. 5a). The syncytium cells were designated STB-(2D) cells. Immunostaining for E-cadherin clearly showed that the STB-(2D) cells were multinuclear (Fig. 5b). The STB marker hCG was highly expressed in these multinuclear syncytia (Fig. 5b). In addition, we observed that higher cell densities were associated with greater numbers of syncytia. The ELISA results showed an increase in hCG levels in 5-day plate culture (Fig. 5c).

Previous studies have reported that cAMP enhances STB formation [32]. Therefore, we treated TS ${ }^{\text {hiPSC }}$ cells with a cAMP agonist, forskolin. However, unlike hTSCs derived from placenta tissue [10], these cells did not effectively fuse to form large syncytia in iMatrix-511-coated dishes. Notably, 3D culture has been reported to enhance the differentiation of trophoblast cells into STB-like cells [8]. Thus, we cultured TS ${ }^{\text {hiPSC }}$ cells in low-adhesion Petri dishes and observed that the cells formed cyst-like cells after 6 days of differentiation (Fig. 5d). The immunostaining results showed the expression of hCG in the cysts (Fig. 5e, f). These cyst-like cells were designated STB-(3D) cells. Based on the qRT-PCR analysis, the expression levels of the hTSCs markers TP63, KRT7, and ELF5 decreased, and those of STB-related markers CGB, MYC, $K L F 4, A B C B 1$, and syndecan 1 (SDC1) increased in STB(3D) cells (Fig. 5g). The ELISA results showed that the levels of hCG secreted by STB-(3D) cells were significantly higher than those secreted by TS ${ }^{\text {hiPSC }}$ cells (Fig. 5 h). The 


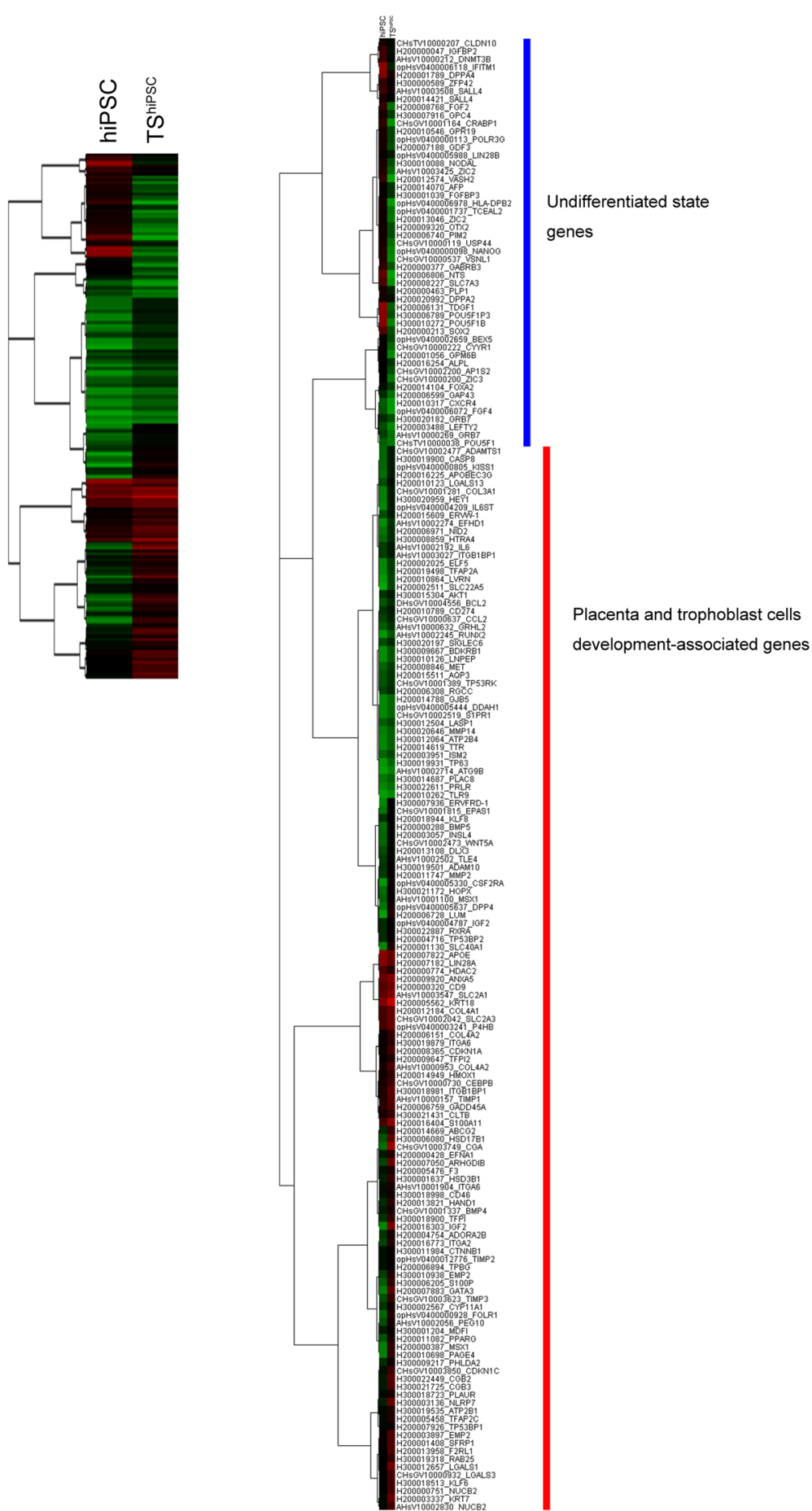

Fig. 4 Comparison of gene signatures between hiPSCS and TS hiPSC cells. Hierarchical clustering heat-map based on the expression of mRNA extracted from hiPSCs and TS ${ }^{\text {hiPSC }}$ cells. Right heat-map including gene names. Green, downregulated genes; red, upregulated genes

differentiation capacity of $\mathrm{TS}^{\text {hiPSC }}$ cells into STB-like cells was demonstrated at passage 59 (Additional file 5: Figure S2A-C).

In addition, we examined the differentiation ability of TS $^{\text {hiPSC }}$ cells into EVT-like cells using a Matrigel-supplemented medium, as described above. The differentiated cells became morphologically different on day 6 (Fig. 5i). The presence of pseudopodia and a mesenchyme-like morphology were noted in these cells (Fig. 5i). HLA-G expression is a known marker for the identification of EVT subpopulations [33]. Immunostaining confirmed HLA-G expression in these differentiated cells (Fig. 5j). 


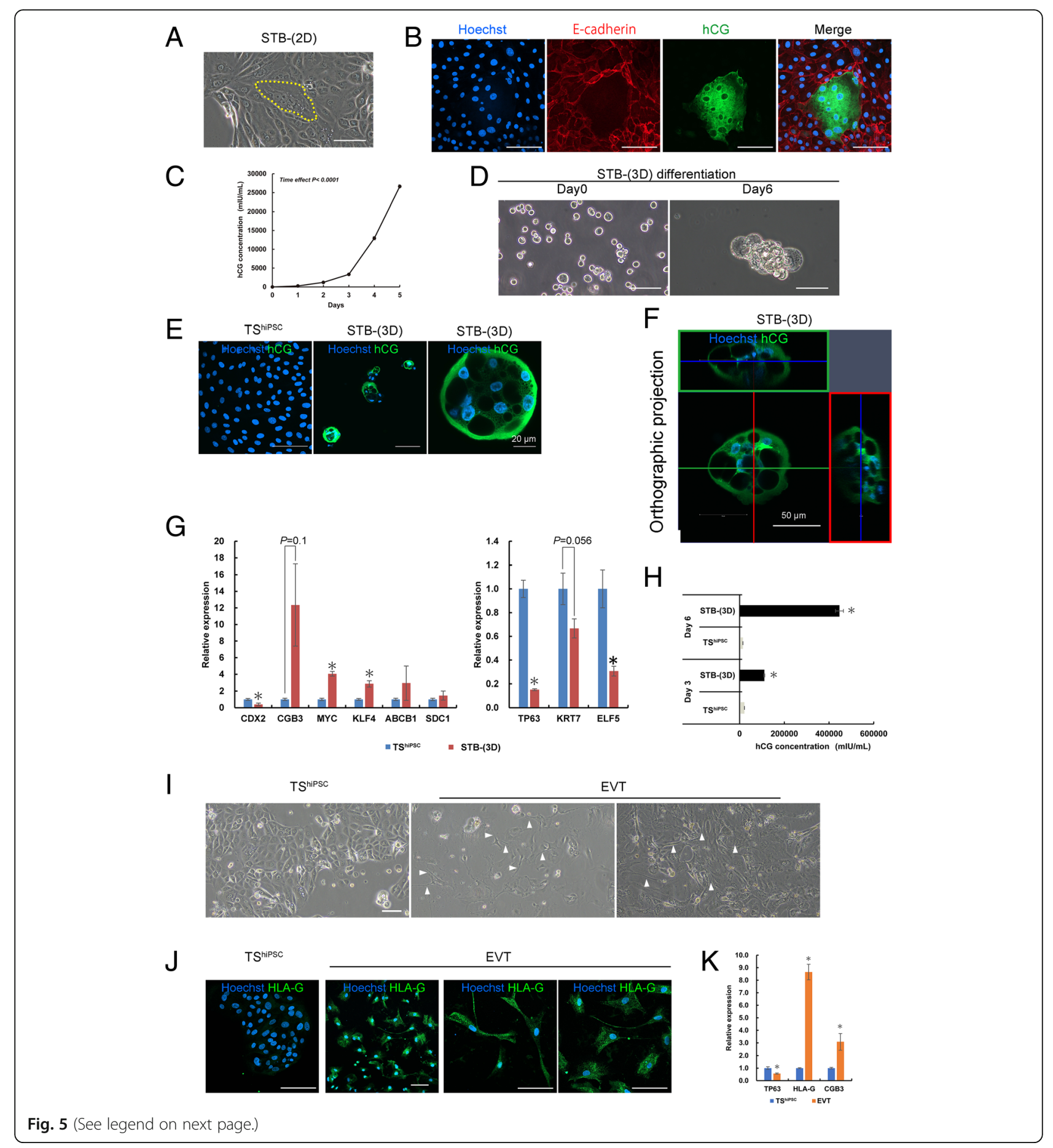


(See figure on previous page.)

Fig. 5 Directed differentiation of TS ${ }^{\text {hiPSC }}$ cells into STB- and EVT-like cells. a Phase-contrast image of TS hiPSC cells containing multinucleated STB(2D) cells (yellow-dotted line area). $\mathbf{b}$ Immunofluorescence images of TS ${ }^{\text {hiPSC }}$ cells containing multinucleated STB-(2D) cells stained for E-cadherin and hCG; nuclei were stained with Hoechst 33342. c Changes in hCG secretion by TS hiPSC cells over 5 days. Data are presented as means \pm SEMs $(n=4)$. Significance of differences was determined by one-way analysis of variance with repeated measures. $\mathbf{d}$ Phase-contrast images of STB-(3D) cells in low-adherence culture dishes on days 0 and 6. e Immunofluorescence images of TS ${ }^{\text {hiPSC }}$ cells and STB-(3D) cells stained for hCG. f Immunofluorescence images of STB-(3D) cells at z-position, showing the STB-(3D) cyst structure, which had several clear interior cavities and a thin enclosing wall. $\mathbf{g}$ Analysis of pluripotency and trophoblast gene expression by qRT-PCR in TShiPSC and STB-(3D) cells. Expression levels were calculated relative to those of GAPDH and normalized to those of TShiPSC cells. Values are the means \pm SEMs $(n=4)$. Significance of differences was determined by Student's $t$ tests. ${ }^{*} P<0.05$ versus the TS ${ }^{\text {hiPSC }}$ cell group. $\mathbf{h}$ Levels of hCG secreted by TS ${ }^{\text {hiPSC }}$ and STB-(3D) cells. For comparison, both TS ${ }^{\text {hiPSC }}$ and STB-(3D) cells were cultured in low-adherence Petri dishes. Data are presented as means \pm SEMs $(n=3)$. i Phasecontrast images of TShiPSC and EVT cells. EVT cells had a mesenchyme-like morphology. The arrows indicate the presence of pseudopodia on EVT cells. $\mathbf{j}$ Immunofluorescence images of TS ${ }^{\text {hiPSC }}$ and EVT cells stained for HLA-G. $\mathbf{k}$ Analysis of TP63, HLA-G, and CGB gene expression by qRT-PCR in $T_{\text {TS }}^{\text {hiPSC }}$ and EVT cells. Expression levels were calculated relative to those of GAPDH and normalized to those in TShiPSC cells. Values are the means \pm SEMs $(n=4)$. Significance of differences was determined by Student's $t$ tests. ${ }^{*} P<0.05$ versus the TS hiPSC cell group. Unless otherwise noted, the scale bars in all phase-contrast and immunostaining images are $100 \mu \mathrm{m}$

Moreover, the qRT-PCR results showed that the human TS marker TP63 decreased, whereas HLA-G and $C G B$ expression levels were significantly increased in these differentiated cells compared with their expression in TS $^{\text {hiPSC }}$ cells (Fig. 5k). Taken together, based on both marker expression and function, these data confirmed the bipotential differentiation ability of $\mathrm{TS}^{\text {hiPSC }}$ cells.

\section{Discussion}

In this study, we successfully purified proliferative CTBlike cells derived from trophectoderm-like cysts, which were induced using a micromesh culture technique, and characterized their ability to proliferate and differentiate as TSCs. Lee et al. [26] suggested that the expression levels of GATA3 and KRT7 proteins should be evaluated as robust criteria for the identification of human first-trimester mononuclear trophoblasts. Importantly, forced expression of TP63 maintains the undifferentiated state of primary CTBs isolated from human first-trimester placental tissues [1]. TP63 expression is significantly downregulated in the later trophoblast lineages EVTs and STBs [1, 10]. Furthermore, both HLA-G and hCG are known to define the two main trophoblast differentiation pathways, i.e., EVTs and STBs, respectively [26]. Our TS ${ }^{\text {hiPSC }}$ cells met all the above criteria and had the ability to directly differentiate into HLA-G-positive EVTs or hCG-secreting STBs. Our comprehensive data strongly suggested that $\mathrm{TS}^{\text {hiPSC }}$ cells may reproduce the features of early hTSCs.

Previous reports by Okeyo et al. [19, 20] have shown that limited culture on micromesh can induce hiPSCs to differentiate into trophoblast cysts. Unlike the studies by Okeyo et al. $[19,20]$, in this study, we used a commercially available nickel micromesh for the differentiation of hiPSCs. In addition, hiPSC lines and their maintenance media were commercially available. Consequently, we showed the ability of hiPSCs to differentiate into
hCG-secreting cysts on the nickel micromesh. Although Okeyo et al. reported that micromesh culture could induce the formation of hCG-secreting cysts, there have been no detailed analyses of the types of cells constituting cysts.

hCG is the most commonly used marker for the identification of STBs [26, 27], and expression of hCG can be used to indicate the presence of STBs in cysts. Immunostaining for E-cadherin showed cell/cell contacts between individual cells in cysts, demonstrating that cysts were primarily composed of mononuclear cells. Thus, we propose that when the micromesh culture method is used, hiPSCs first differentiate into mononuclear CTBs, and these CTBs then further differentiate into STBs in the 3D cystic structure. Interestingly, CTBlike cells were proliferative in hiPSC maintenance medium.

Studies of trophoblast differentiation from hiPSCs and hESCs have focused on the use of BMP4. Short-term (2-6 days) and high-dose $(10 \mathrm{ng} / \mathrm{mL})$ BMP4 treatment has been shown to induce a trophoblast lineage [15]. BMP4-induced trophoblast cells often do not really resemble primary CTBs because the composition of the culture medium is inadequate. Recently, two groups have improved BMP4 treatment conditions and confirmed the generation of CDX2-, TP63-, and KRT7positive CTB populations $[1,15]$. These cells could further differentiate into STBs and EVTs upon BMP4 treatment $[1,15]$. However, these BMP4-treated CTBs did not have proliferative ability, and their directional differentiation has not been tested [15]. In this study, we used marker analysis, molecular pattern analysis, and secretory function to confirm that the phenotype of TS ${ }^{\text {hiPSC }}$ cells was similar to that of BMP4-treated CTBs. In our study, hiPSC-derived proliferative and bipotential CTB-like cells were obtained for the first time. The TS ${ }^{\text {hiPSC }}$ cells, as a primary CTB model, were superior to BMP4-treated CTB-like cells. 
Identification of BMP4-induced trophoblasts has focused on the induction of CDX2, a transcription factor proposed to be essential for trophectoderm identity and maintenance [34]. CDX2 is detectable until the trophectoderm can be morphologically distinguished and cannot be detected at the morula and early blastocyst stages ( day 4$)$. CDX2 is then downregulated and translocated to the cytoplasm in human peri-implantation embryos (day 8) [35]. CDX2 is expressed in the human placenta during early gestation (6 weeks) [15] but is expressed at very low levels in hTSCs established from the first-trimester placenta ( 14 weeks) [10]. Our results showed that expression of the CDX2 gene was 9-fold higher in trophectoderm-like cysts than in hiPSCs, whereas its level of expression in TS ${ }^{\text {hiPSC }}$ cells was only approximately $10 \%$ of that in hiPSCs. Moreover, immunostaining results showed that CDX2 protein was barely expressed both in hiPSCs and TS ${ }^{\text {hiPSC }}$ cells (Additional file 4: Figure S1C). Quantitative results from immunostaining analyses showed that there were no significant differences between hiPSCs and TS ${ }^{\text {hiPSC }}$ cells (Additional file 4: Figure S1D). These results could be related to the observation that some cyst cells were in a differentiated state. When the medium was changed from the hiPSC medium to the hTSC medium, purified TS ${ }^{\text {hiPSC }}$ cells showed a homogeneous phenotype, which was similar to that of hTSCs from blastocysts and the first-trimester placenta $\left(\mathrm{TS}^{\text {placenta }}\right)$ with low $\mathrm{CDX} 2$ expression [10].

Bipotent CTB subtypes are only present in the first-trimester placenta and are lost during the second trimester of pregnancy [36]. CTBs derived from the first-trimester placenta are highly proliferative and have the capacity to generate new villi; these CTBs were suggested to serve as the source of hTSCs [37]. Recently, Okae et al. [10] have successfully isolated and established $\mathrm{TS}^{\text {placenta }}$. The use of tissues from human embryos and the first-trimester placenta is a good strategy; however, the tissues are often difficult to obtain because of moral and ethical issues. TS $^{\text {placenta }}$ cells can proliferate and differentiate into EVTand STB-like cells [10]. Our TS ${ }^{\text {hiPSC }}$ cells, unlike conventional BMP4-treated CTB-like cells, maintained the properties of $\mathrm{TS}^{\text {placenta }}$ cells. Regarding their ability to differentiate, TS $^{\text {placenta }}$ cells can fuse into STBs in 2D culture dishes upon forskolin stimulation. TS ${ }^{\text {hiPSC }}$ cells do not efficiently fuse into STBs in 2D culture, but can fuse into 3D-STBs in low-adherence culture. This fusion ability may depend on the adherence ability of TS ${ }^{\text {hiPSC }}$ cells. Further studies are needed to determine the fusion conditions for $\mathrm{TS}^{\text {hiPSC }}$ cells in 2D culture.

In this study, transcriptome analysis was performed on $\mathrm{TS}^{\text {hiPSC }}$ cells and hiPSCs, and our future research will focus on further identification of differences between $\mathrm{TS}^{\text {hiPSC }}$ cells and primary trophoblast cells. The ChiPSC22 line used in this study and the hiPSCs (clone TIG1-4F hiPSC from lung fibroblasts, provided by Dr. Takashi Tada of Kyoto University, Japan), which were reported by Okeyo et al. [19, 20], have been shown to be able to differentiate into trophectoderm under micromesh culture. Furthermore, we also found that differentiation into trophectoderm was achieved by spontaneous selforganization, which may or may not occur with similar efficiencies in other hiPSC lines. We used $201 \mathrm{~B} 7$ and 253G1 hiPSC lines. The 201B7 line only could differentiate to obtain very few cysts (data not shown). The 253G1 line could not differentiate into cysts under the micromesh culture (data not shown).

It is important to determine why the differentiation ability into the trophoblast lineage was different between hiPSCs for application of the micromesh culture technique in the cell differentiation induction field. We also speculated that the production method of hiPSCs, hiPSC properties, and medium components may affect the ability of the cells to differentiate. In the future, we will induce differentiation into trophoblast cells with multiple hiPSC strains and clarify the factors that modulate the induction of differentiation into trophoblast lineages by micromesh culture at a molecular level.

Overall, compared with previous studies on the differentiation of trophoblast cells from hiPSCs, we obtained hTSC-like cells derived from hiPSCs, for the first time. This differentiation process was performed without using BMP4. Therefore, we expect that this simple, versatile micromesh culture technique may have potential applications in the differentiation of iPSCs into other cell types. Horii et al. [15] reported a two-step protocol for obtaining CTB stem-like cells from hiPSCs. However, these CTB stem-like cells were not proliferative, and their redifferentiation could not be controlled directionally. Thus, we propose that, except in primary cells, our micromesh culture protocol may be superior to other currently available models for studying TSCs.

\section{Conclusions}

In summary, we established hTSC-like cells derived from hiPSCs, which were induced using a micromesh technique without any chemical stimulation. These established cells represent a novel, powerful model for studying the function and molecular characteristics of human trophoblast cells. This approach also provided novel insights into the mechanisms of hiPSC differentiation into trophoblast cells and a new experimental system for studying the steps of human trophoblast differentiation. The experimental system may provide a useful tool for understanding the pathogenesis of developmental disorders with trophoblast defects, such as preeclampsia, miscarriage, and intrauterine growth restriction. 


\section{Additional file}

Additional file 1: Table S1. Markers and primer sequences used for real-time qRT-PCR analysis. (DOCX $15 \mathrm{~kb}$ )

Additional file 2: Table S2. Global gene expression profiling data. (XLSX $106 \mathrm{~kb}$ )

Additional file 3: Movie S1. A single cyst was isolated from a micromesh culture on day 46 of culture. (MP4 3870 kb)

Additional file 4: Figure S1. Trophoblast marker expression in trophoblast cells derived from cysts (TS ${ }^{\text {hiPSC}}$ ) after prolonged cell culture. (A) Immunofluorescence images of hiPSCs and TS ${ }^{\text {hiPSC }}$ cells stained for KRT7, TP63, and GATA3. Nuclei were stained with Hoechst 33342. Scale bar $=20 \mu \mathrm{m}$. (B) Analysis of pluripotency and trophoblast gene expression by qRT-PCR in hiPSCs and TS hiPSC cells. Expression levels were calculated relative to those of GAPDH and normalized to those of control hiPSCs. The relative expression levels were also log-transformed. Values are the means \pm SEMs $(n=4)$. (C) Immunofluorescence images of hiPSCs and TS $^{\text {hiPSC }}$ cells stained for CDX2. Scale bar $=20 \mu \mathrm{m}$. (D) Quantitative results of CDX2 expression from immunostaining analyses. Values are the means \pm SEMs $(n=6)$. (TIF $6182 \mathrm{~kb})$

Additional file 5: Figure S2. Directed differentiation of TS ${ }^{\text {hiPSC }}$ cells (P59) into STB-(3D) cells. (A) Phase-contrast images of TS ${ }^{\text {hiPSC }}$ and STB(3D) cells on days 0,3 , and 5. For comparison, both TS ${ }^{\text {hiPSC }}$ and STB-(3D) cells were cultured in low-adherence Petri dishes. (B) High-magnification phase-contrast and stereomicroscope images of STB-(3D) cells on day 5. (C) Immunofluorescence images of TS ${ }^{\text {hiPSC }}$ and STB-(3D) cells on day 6. The cells were stainted for hCG, and the nuclei were stained with Hoechst 33342. Scale bar $=100 \mu \mathrm{m}$. (TIF 8908 kb)

\section{Abbreviations}

ABCB1: ATP-binding cassette subfamily B member 1; BMP4: Bone morphogenetic protein-4; CAMP: Cyclic adenosine monophosphate; CDX2: Caudal type homeobox 2; CGB3: Chorionic gonadotropin subunit beta 3; CTBs: Mononuclear cytotrophoblasts; ELF5: E74-like ETS transcription factor 5; EVTs: Extravillous trophoblasts; FGF2: Fibroblast growth factor-2; GAPDH: Glyceraldehyde 3-phosphate dehydrogenase; GATA3: GATA-binding protein 3; hCG: Human chorionic gonadotrophin; hESCs: Human embryonic stem cells; hiPSCs: Human induced pluripotent stem cells; HLA-G: Major histocompatibility complex, class I, G; hTSCs: Human trophoblast stem cells; KLF4: Kruppel-like factor 4; KRT7: Keratin 7; NANOG: Nanog homeobox; PFA: Paraformaldehyde; POU5F1: POU class 5 homeobox 1; qRT-PCR: Realtime quantitative polymerase chain reaction; SOX2: SRY-box 2; STB: Syncytiotrophoblast; TP63: Tumor protein p63; TSCs: Trophoblast stem cells; TS hiPSC: TSCs from hiPSC-induced cysts

\section{Acknowledgements}

We thank Dr. T. Hori, Ms. H. Ito, and Mr. N. Tani for the support and technical assistance and Prof. M. Washizu of the University of Tokyo and Prof. K.O. Okeyo of Kyoto University for the valuable suggestions.

\section{Authors' contributions}

ZL contributed to the conception and design of the study, collection and/or assembly of the data, data analysis and interpretation, and manuscript writing. OK and $\mathrm{HI}$ contributed to the conception and design of the study and obtained financial support. All authors read and approved the final manuscript.

\section{Funding}

This research was supported by grants from the Japan Society for the Promotion of Science (JSPS) (KAKENHI grant no.: 18 K12071) and the "Compass to Healthy Life" Research Complex Program of the Japan Science and Technology Agency (JST).

\section{Availability of data and materials}

The datasets used and/or analyzed during the current study are available from the corresponding author on reasonable request.

\section{Ethics approval and consent to participate}

Not applicable.
Consent for publication

Not applicable.

\section{Competing interests}

The authors declare that they have no competing interests.

\section{Author details}

"The "Compass to Healthy Life" Research Complex Program, RIKEN Institute, Kobe 650-0047, Japan. ${ }^{2}$ Research Promotion Institution for COI Site, Kyoto University, Kyoto, Japan.

Received: 20 March 2019 Revised: 26 June 2019

Accepted: 14 July 2019 Published online: 07 August 2019

\section{References}

1. Li Y, Moretto-Zita M, Soncin F, Wakeland A, Wolfe L, Leon-Garcia S, et al. BMP4-directed trophoblast differentiation of human embryonic stem cells is mediated through a $\Delta N p 63+$ cytotrophoblast stem cell state. Development. 2013;140:3965-76.

2. Yabe S, Alexenko AP, Amita M, Yang Y, Schust DJ, Sadovsky Y, et al. Comparison of syncytiotrophoblast generated from human embryonic stem cells and from term placentas. Proc Natl Acad Sci U S A. 2016; 113:E2598-E607.

3. Bansal AS, Bora SA, Saso S, Smith JR, Johnson MR, Thum MY. Mechanism of human chorionic gonadotrophin-mediated immunomodulation in pregnancy. Expert Rev Clin Immunol. 2012;8:747-53.

4. Huppertz B, Meiri H, Gizurarson S, Osol G, Sammar M. Placental protein 13 (PP13): a new biological target shifting individualized risk assessment to personalized drug design combating pre-eclampsia. Hum Reprod Update. 2013;19:391-405.

5. Fujiwara $H$, Higuchi T, Sato $Y$, Nishioka $Y$, Zeng BX, Yoshioka S, et al. Regulation of human extravillous trophoblast function by membrane-bound peptidases. Biochim Biophys Acta. 2005;1751:26-32.

6. Hemberger M. Health during pregnancy and beyond: fetal trophoblast cells as chief co-ordinators of intrauterine growth and reproductive success. Ann Med. 2012:44:325-37.

7. Wang R, Dang YL, Zheng R, Li Y, Li W, Lu X, et al. Live cell imaging of in vitro human trophoblast syncytialization. Biol Reprod. 2014;90:117.

8. McConkey CA, Delorme-Axford E, Nickerson CA, Kim KS, Sadovsky Y, Boyle JP, et al. A three-dimensional culture system recapitulates placental syncytiotrophoblast development and microbial resistance. Sci Adv. 2016:2:e1501462.

9. Kliman HJ, Nestler JE, Sermasi E, Sanger JM, Strauss JF 3rd. Purification, characterization, and in vitro differentiation of cytotrophoblasts from human term placentae. Endocrinology. 1986;118:1567-82.

10. Okae H, Toh H, Sato T, Hiura H, Takahashi S, Shirane K, et al. Derivation of human trophoblast stem cells. Cell Stem Cell. 2018;22:50-63 e6.

11. Schulz LC, Ezashi T, Das P, Westfall SD, Livingston KA, Roberts RM. Human embryonic stem cells as models for trophoblast differentiation. Placenta. 2008;29:S10-S6

12. Amita M, Adachi $K$, Alexenko AP, Sinha S, Schust DJ, Schulz LC, et al. Complete and unidirectional conversion of human embryonic stem cells to trophoblast by BMP4. Proc Natl Acad Sci U S A. 2013;110:E1212-E21.

13. Sudheer S, Bhushan R, Fauler B, Lehrach H, Adjaye J. FGF inhibition directs BMP4-mediated differentiation of human embryonic stem cells to syncytiotrophoblast. Stem Cells Dev. 2012;21:2987-3000.

14. Chen Y, Wang K, Chandramouli GVR, Knott JG, Leach R. Trophoblast lineage cells derived from human induced pluripotent stem cells. Biochem Biophys Res Commun. 2013;436:677-84.

15. Horii M, Li YC, Wakeland AK, Pizzo DP, Nelson KK, Sabatini K, et al. Human pluripotent stem cells as a model of trophoblast differentiation in both normal development and disease. Proc Natl Acad Sci U S A. 2016;113:E3882-E91.

16. Wang YY, Hu J, Jiao J, Liu ZN, Zhou Z, Zhao C, et al. Engineering vascular tissue with functional smooth muscle cells derived from human iPS cells and nanofibrous scaffolds. Biomaterials. 2014:35:8960-9.

17. Bilousova G, Jun DH, King KB, De Langhe S, Chick WS, Torchia EC, et al. Osteoblasts derived from induced pluripotent stem cells form calcified structures in scaffolds both in vitro and in vivo. Stem Cells. 2011;29:206-16. 
18. Hori T, Kurosawa O. A three-dimensional cell culture method with a micromesh sheet and its application to hepatic cells. Tissue Eng Part C Methods. 2018;24:730-9.

19. Okeyo KO, Tanabe M, Kurosawa O, Oana H, Washizu M. Self-organization of human iPS cells into trophectoderm mimicking cysts induced by adhesion restriction using microstructured mesh scaffolds. Develop Growth Differ. 2018;60:183-94

20. Okeyo KO, Kurosawa O, Yamazaki S, Oana H, Kotera H, Nakauchi H, et al. Cell adhesion minimization by a novel mesh culture method mechanically directs trophoblast differentiation and self-assembly organization of human pluripotent stem cells. Tissue Eng Part C Methods. 2015;21:1105-15.

21. Fan J, Wang M, Wang C, Cao Y. Advances in human chorionic gonadotropin detection technologies: a review. Bioanalysis. 2017;9:1509-29.

22. Ma GT, Roth ME, Groskopf JC, Tsai FY, Orkin SH, Grosveld F, et al. GATA-2 and GATA-3 regulate trophoblast-specific gene expression in vivo. Development. 1997;124:907-14.

23. Peng $L H$, Huang $Y$, Jin $F$, Jiang SW, Payne AH. Transcription enhancer factor5 and a GATA-like protein determine placental-specific expression of the type I human 3ß-hydroxysteroid dehydrogenase gene, HSD3B1. Mol Endocrinol. 2004;18:2049-60.

24. Constancia M, Hemberger M, Hughes J, Dean W, Ferguson-Smith A, Fundele $\mathrm{R}$, et al. Placental-specific IGF-II is a major modulator of placental and fetal growth. Nature. 2002;417:945-8.

25. Tian H, Hammer RE, Matsumoto AM, Russell DW, McKnight SL. The hypoxia-responsive transcription factor EPAS1 is essential for catecholamine homeostasis and protection against heart failure during embryonic development. Genes Dev. 1998;12:3320-4.

26. Lee CQ, Gardner L, Turco M, Zhao N, Murray MJ, Coleman N, et al. What is trophoblast? A combination of criteria define human first-trimester trophoblast. Stem Cell Rep. 2016;6:257-72.

27. Genbacev O, Donne M, Kapidzic M, Gormley M, Lamb J, Gilmore J, et al. Establishment of human trophoblast progenitor cell lines from the chorion. Stem Cells. 2011:29:1427-36.

28. Xu RH, Chen X, Li DS, Li R, Addicks GC, Glennon C, et al. BMP4 initiates human embryonic stem cell differentiation to trophoblast. Nat Biotechnol. 2002;20:1261-4.

29. Lichtner B, Knaus P, Lehrach H, Adjaye J. BMP10 as a potent inducer of trophoblast differentiation in human embryonic and induced pluripotent stem cells. Biomaterials. 2013;34:9789-802.

30. Marchand M, Horcajadas JA, Esteban FJ, McElroy SL, Fisher SJ, Giudice LC. Transcriptomic signature of trophoblast differentiation in a human embryonic stem cell model. Biol Reprod. 2011;84:1258-71.

31. Wu Z, Zhang W, Chen GB, Cheng L, Liao J, Jia N, et al. Combinatorial signals of activin/nodal and bone morphogenic protein regulate the early lineage segregation of human embryonic stem cells. J Biol Chem. 2008;283:24991-5002

32. Strauss JF, Kido S, Sayegh R, Sakuragi N, Gafvels ME. The CAMP signaling system and human trophoblast function. Placenta. 1992;13:389-403.

33. Apps R, Murphy SP, Fernando R, Gardner L, Ahad T, Moffett A. Human leucocyte antigen (HLA) expression of primary trophoblast cells and placental cell lines, determined using single antigen beads to characterize allotype specificities of anti-HLA antibodies. Immunology. 2009;127:26-39.

34. Piliszek A, Grabarek JB, Frankenberg SR, Plusa B. Cell fate in animal and human blastocysts and the determination of viability. Mol Hum Reprod. 2016;22:681-90

35. Chen AE, Egli D, Niakan K, Deng J, Akutsu H, Yamaki M, et al. Optimal timing of inner cell mass isolation increases the efficiency of human embryonic stem cell derivation and allows generation of sibling cell lines. Cell Stem Cell. 2009:4:103-6.

36. James JL, Carter AM, Chamley LW. Human placentation from nidation to 5 weeks of gestation. Part II: tools to model the crucial first days. Placenta. 2012;33:335-42.

37. Castellucci M, Kosanke G, Verdenelli F, Huppertz B, Kaufmann P. Villous sprouting: fundamental mechanisms of human placental development. Hum Reprod Update. 2000;6:485-94.

\section{Publisher's Note}

Springer Nature remains neutral with regard to jurisdictional claims in published maps and institutional affiliations.

\section{Ready to submit your research? Choose BMC and benefit from:}

- fast, convenient online submission

- thorough peer review by experienced researchers in your field

- rapid publication on acceptance

- support for research data, including large and complex data types

- gold Open Access which fosters wider collaboration and increased citations

- maximum visibility for your research: over $100 \mathrm{M}$ website views per year

At BMC, research is always in progress.

Learn more biomedcentral.com/submissions 